 \\ Respiratory and Pulmonary Medicine
}

\section{Correlation between Pentraxin-3 and Left Ventricular Diastolic Dysfunction in Obstructive Sleep Apnea}

\author{
Jie-Ru Li', Xiu-Hua Gao ${ }^{2}$, Ju-Qiang Han ${ }^{3}$, Wen-Yan Luo and En-Sheng Ji ${ }^{1^{*}}$ \\ ${ }^{1}$ Department of Physiology, Hebei University of Chinese Medicine, People's Republic of China \\ ${ }^{2}$ Department of Respiratory Medicine, Chinese People's Liberation Army Bethune International Peace Hospital, People's \\ Republic of China
}

${ }^{3}$ Department of Surgical Nursing, Hebei University of Chinese Medicine, People's Republic of China

${ }^{4}$ Department of Pathobiology, Hebei University of Chinese Medicine, People's Republic of China

*Corresponding author: En-sheng Jl, Department of Physiology, Hebei University of Chinese Medicine, Luquan Xingyuan Road 3, Shijiazhuang, Hebei 050200, People's Republic of China, Tel: 0311-89926098

\begin{abstract}
Objective: Obstructive sleep apnea (OSA) is one of independent dangerous factors for cardiovascular disease including heart failure. Recent studies demonstrated that left ventricular diastolic dysfunction (LVDD) and pentraxin-3 (PTX-3) are early signs for development of heart failure. The objective of our study was to estimate the relation between OSA severity and LVDD and PTX-3.

Methods: Sixty-six OSA patients and 25 healthy people who underwent polysomnography to diagnose OSA were enrolled. Ninety-one participants were divided into three groups as per apnea-hypopnea index (AHI), including mild $(5 \leq \mathrm{AHI}<15)$ and moderate-severe OSA $(\mathrm{AHI} \geq 15)$ and control group $(\mathrm{AHI}<5)$. Left ventricular diastolic function was evaluated by the ratio of early diastolic peak transmitral flow velocity $(E) / p e a k$ early diastolic mitral annular velocity $(E m)(E / E m)$ and maximal left atrial volume index (LAVI). Peripheral blood levels of PTX-3 were measured.

Results: Our results showed that levels of PTX-3 increased significantly, and the ratio of E/Em and maximal LAVI increased obviously with the increase of OSA severity. There was a remarkable positive correlation between the $\mathrm{E} /$ Em ratio, maximal LAVI and AHI. Levels of PTX-3 in serum were positively correlated with the $\mathrm{E} / \mathrm{Em}$ ratio. Levels of PTX-3 were also positively related with maximal LAVI.

Conclusion: OSA patients showed significant enhancement of PTX-3 levels and LVDD. Furthermore, both PTX-3 and LVDD were independently correlated with severity of OSA defined by AHI. Serum PTX-3 levels were positively correlated with LVDD in OSA patients.
\end{abstract}

\section{Keywords}

Left ventricular diastolic dysfunction, E/Em, Left atrial volume index, Obstructive sleep apnea, Pentraxin-3

\section{Introduction}

Obstructive sleep apnea (OSA) is a frequent sleeprelated respiratory disease with frequent upper airway total or partial obstruction during sleep [1]. Chronic intermittent hypoxia $(\mathrm{ClH})$ and hypoxemia attributing to apnea provoke systemic effects and increase levels of biomarkers related to cardiovascular abnormality [2].

Left ventricular diastolic dysfunction (LVDD) is an early marker of cardiac function abnormality before clinically noticeable cardiovascular disease $[3,4]$. It is characterized by reducing and/or totally losing the relaxation and compliance of cardiomyocytes. Echocardiographic examination is used as a convenient and reliable method to measure left ventricular functions. Diastolic function was evaluated by the ratio of early diastolic peak transmitral flow velocity $(E) /$ peak early diastolic mitral annular velocity (Em) and left atrial volume index (LAVI) [5]. Early diagnosis and effective intervention of LVDD have become one of the most pressing problems in the cardiovascular field.

Systemic inflammation is increasingly identified as a risk factor for some cardiovascular complications

Citation: Jie-Ru L, Xiu-Hua G, Ju-Qiang H, Wen-Yan L, En-Sheng J (2018) Correlation between Pentraxin-3 and Left Ventricular Diastolic Dysfunction in Obstructive Sleep Apnea. Int J Respir Pulm Med 5:097. doi.org/10.23937/2378-3516/1410097

Accepted: November 21, 2018; Published: November 23, 2018

Copyright: (C) 2018 Jie-Ru L, et al. This is an open-access article distributed under the terms of the Creative Commons Attribution License, which permits unrestricted use, distribution, and reproduction in any medium, provided the original author and source are credited 
[6] and an acceptable factor in the pathogenesis of cardiovascular disorders in patients with co-existent OSA $[2,7]$. Inflammatory markers are closely concerned with the pathogenesis of heart failure, adverse prognoses and the worst cardiac function. Pentraxin-3 (PTX-3) was recently identified as one of the members of the pentraxin family, including C-reactive protein (CRP) and serum amyloid P. PTX-3 was generated by endothelial cells, monocyte-macrophages and neutrophils following inflammatory stimuli [8]. Compared with hs-CRP, PTX-3 might definitely and sensitively reflect localized inflammation in tissues and was regarded as a novel biomarker of cardiovascular system inflammation [9]. Recent studies showed that serum PTX-3 levels significantly increased in moderate and severe OSA patients $[9,10]$, and PTX-3 was an independent inflammatory marker associated with the occurrence of LVDD [4,11]. However, the relationship between PTX-3 and LVDD remained unclear in OSA patients. The objective of the present study was to appraise the correlation between inflammatory markers and LVDD among OSA patients.

\section{Subjects and Methods}

\section{Study population}

A cross-sectional study was conducted in Department of Respiratory Medicine of Chinese People's Liberation Army Bethune International Peace Hospital. The study protocol was authorized by ethics committee for clinical research in Chinese People's Liberation Army Bethune International Peace Hospital. All subjects offered informed consent in writing.

Ninety-one suspected OSA patients presenting to Department of Respiratory Medicine in the present hospital who finished overnight polysomnography (PSG) were enlisted in our study. Patients with smoking history, active infections, chronic obstructive pulmonary disease, hypertension, diabetes mellitus, left ventricular systolic dysfunction (defined as left ventricular ejection fraction (LVEF) $<50 \%$ ) and heart disease were removed from our study. Subjects with apnea hypopnea index $(\mathrm{AHI})<5$ were regarded as control group. The $\mathrm{AHI}$ cutoff point of diagnosis for mild OSAS was 5-15, and moderate-severe OSA was diagnosed by $\mathrm{AHI} \geq 15$ incidents in an hour of sleep.

\section{PSG}

Overnight PSG was performed in subjects from 22 p.m. to 6 a.m., and parameters were recorded using a computer system (Alice 4, Philips Respironics, Murrysville, PA, USA). Apnea, hypopnea, and electroencephalogram recordings were manually graded as per standard criteria. Apnea was considered as a complete Interrupt of nasal flow for at the least 10 second. Hypopnea was defined as a $50 \%$ reduction in airflow for at least 10 second together with at least $4 \%$ of oxyhaemoglobin desaturation or an arousal registered by the electroencephalogram. $\mathrm{AHI}$ was calculated according to the mean number of apnea and hypopnea during PSG-recorded sleep time.

\section{Assessment of left ventricular diastolic function}

Echocardiographic examination was regarded as one of most convenient and reliable methods to assess left ventricular functions. Thus, the day after PSG finished, transthoracic echocardiographic examination using a Philips iE33 ultrasound system and S5-1 broadband phased array transducer was carried out by a cardiologist who knew nothing about the PSG results. Left ventricular diameters, interventricular septum thickness, left ventricular posterior wall thickness, and left atrial diameter were checked by M-mode echocardiography. LVEF was computed by the modified Simpson technique. E and late diastolic peak transmitral flow velocity (A), E/A ratio, and deceleration time were measured with pulsed wave Doppler. Em and peak late diastolic mitral annular velocity $(\mathrm{Am})$ were obtained by pulse-wave tissue Doppler imaging at the septal site of the mitral annulus. The E/Em ratio was used as an index of left ventricular filling pressure. Maximal left atrial volume (LAV) was also measured using the biplane modified Simpson's method, and LAVI was calculated as maximal LAV/body surface area.

\section{Blood collection and biochemical measurements}

After PSG, blood samples were taken from all overnight fasting participants and placed in ethylenediamine tetra acetic acid coated polypropylene tubes. The blood samples were centrifuged by $5000 \mathrm{r} / \mathrm{m}$ for $10 \mathrm{~min}$ at $4{ }^{\circ} \mathrm{C}$, and the clear plasma supernatants were stored at $-80^{\circ} \mathrm{C}$. Plasma PTX-3 was detected by enzyme-linked immunosorbent assay (ELISA) with Quantikine ${ }^{\circledR}$ ELISA (Human PTX-3 Immunoassay, R\&D Systems, Inc., Minneapolis, USA).

\section{Statistical analysis}

Continuous variables were expressed as mean \pm SD. Results were analyzed using one-way ANOVA with least significant difference tests for LSD comparisons, correlations between variables were explored by using Pearson coefficient. $P$ value less than 0.05 was considered as statistical significance. Statistical analysis was carried out by using SPSS statistical software version 19.0 (Chicago, Illinois, USA).

\section{Results}

\section{Clinical characteristics of subjects in this study}

Clinical characteristics of OSA patients and control group in this study were shown in Table 1. No significant differences were shown among 3 groups in age, BMI, total cholesterol (TC), triglycerides (TG), or fasting blood glucose (FBG). However, remarkable differences were observed in $\mathrm{AHI}$ and oxygen desaturation index (ODI) among 3 groups. As expected, ODI and AHI were gradually augmented as the severity of OSA increased (Table 1). 
Table 1: Clinical and metabolic characteristics of patients with OSA and normal control.

\begin{tabular}{|c|c|c|c|c|}
\hline Variables & Control & Mild OSA & Moderate-Severe OSA & $\boldsymbol{P}$ \\
\hline Age (years) & $45.08 \pm 5.54$ & $45.67 \pm 6.02$ & $47.61 \pm 7.35$ & 0.270 \\
\hline BMI (kg/m²) & $26.57 \pm 2.57$ & $26.69 \pm 3.03$ & $27.10 \pm 2.76$ & 0.731 \\
\hline ODI & $1.29 \pm 0.83$ & $9.61 \pm 3.73^{\star}$ & $44.11 \pm 11.50^{* \#}$ & 0.000 \\
\hline $\mathrm{AHI}$ & $1.52 \pm 1.64$ & $10.97 \pm 2.53^{*}$ & $37.64 \pm 13.60^{* \#}$ & 0.000 \\
\hline $\mathrm{TC}(\mathrm{mmol} / \mathrm{l})$ & $3.94 \pm 0.48$ & $4.17 \pm 0.44$ & $4.20 \pm 0.42$ & 0.053 \\
\hline TG $(\mathrm{mmol} / \mathrm{l})$ & $1.29 \pm 0.20$ & $1.41 \pm 0.22$ & $1.32 \pm 0.21$ & 0.116 \\
\hline $\mathrm{FBG}(\mathrm{mmol} / \mathrm{l})$ & $4.94 \pm 0.55$ & $5.02 \pm 0.51$ & $5.21 \pm 0.43$ & 0.068 \\
\hline PTX-3 (ng/ml) & $1.87 \pm 0.73$ & $2.62 \pm 1.15^{\star}$ & $4.90 \pm 1.72^{* \#}$ & 0.000 \\
\hline LVEF (\%) & $62.59 \pm 8.10$ & $61.55 \pm 6.64$ & $59.06 \pm 7.14$ & 0.148 \\
\hline $\mathrm{E}(\mathrm{cm} / \mathrm{s})$ & $75.97 \pm 7.40$ & $75.40 \pm 7.52$ & $74.50 \pm 6.65$ & 0.720 \\
\hline $\mathrm{A}(\mathrm{cm} / \mathrm{s})$ & $70.93 \pm 7.48$ & $71.55 \pm 5.94$ & $74.16 \pm 4.23$ & 0.069 \\
\hline $\mathrm{E} / \mathrm{A}$ & $1.06 \pm 0.12$ & $1.04 \pm 0.08$ & $0.99 \pm 0.09$ & 0.051 \\
\hline $\mathrm{Em}(\mathrm{cm} / \mathrm{s})$ & $10.14 \pm 1.39$ & $8.85 \pm 2.00^{\star}$ & $7.91 \pm 1.51^{* \#}$ & 0.000 \\
\hline $\mathrm{Am}(\mathrm{cm} / \mathrm{s})$ & $9.12 \pm 1.44$ & $9.52 \pm 1.32$ & $9.98 \pm 1.42$ & 0.061 \\
\hline $\mathrm{Em} / \mathrm{Am}$ & $1.06 \pm 0.12$ & $0.95 \pm 0.23^{*}$ & $0.81 \pm 0.14^{* \#}$ & 0.000 \\
\hline $\mathrm{E} / \mathrm{Em}$ & $7.60 \pm 1.12$ & $8.74 \pm 2.18^{\star}$ & $9.71 \pm 2.21^{* \#}$ & 0.000 \\
\hline Max LAVI $\left(\mathrm{ml} / \mathrm{m}^{2}\right)$ & $11.71 \pm 3.07$ & $12.87 \pm 3.86^{*}$ & $17.09 \pm 5.13^{*}$ & 0.000 \\
\hline
\end{tabular}

BMI: Body Mass Index; ODI: Oxygen Desaturation Index; AHI: Apnea Hypopnea Index; TC: Total Cholesterol; TG: Triglyceride; FBG: Fasting Blood Glucose; PTX-3: Pentraxin-3; LVEF: Left Ventricular Ejection Fraction; E: Early Diastolic Peak Transmitral Flow Velocity; A: Late Diastolic Peak Transmitral Flow Velocity; Em: Peak Early Diastolic Mitral Annular Velocity; Am: Peak Late Diastolic Annular Velocity; LAVI: Left Atrial Volume Index. Data are presented as mean \pm SD. $(n=25$ in control group, $n=30$ in mild OSA group, $\mathrm{n}=36$ in moderate-severe OSA group). *: $\mathrm{P}<0.05$, mild OSA and moderate-severe OSA groups vs. control; \#: $\mathrm{P}<0.05$, moderate-severe OSA group vs. mild OSA.

\section{A}
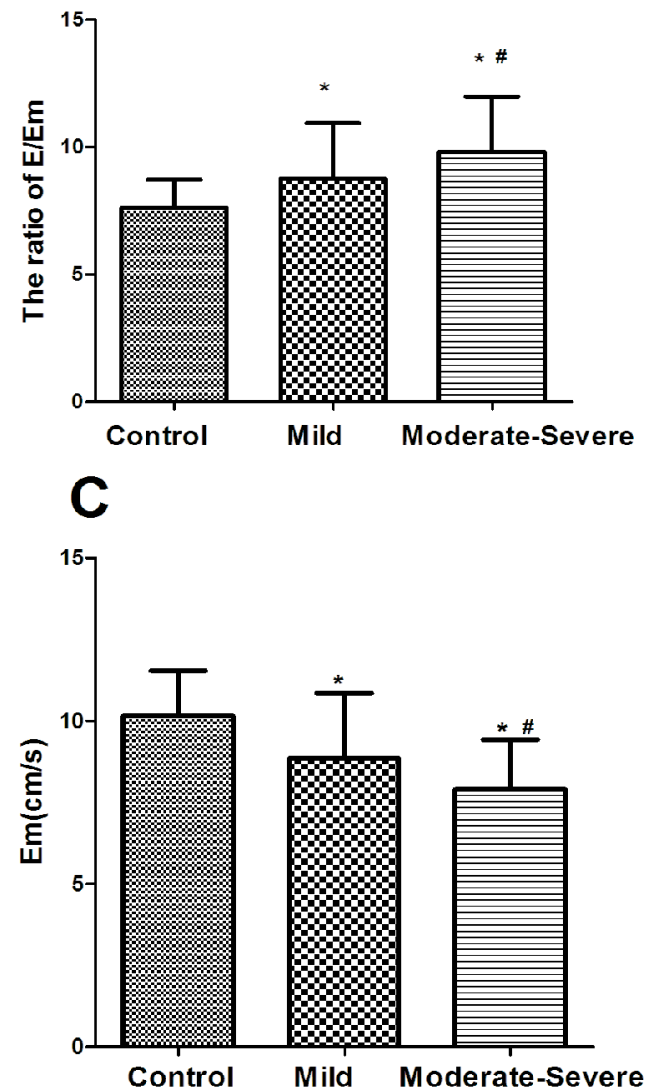

B
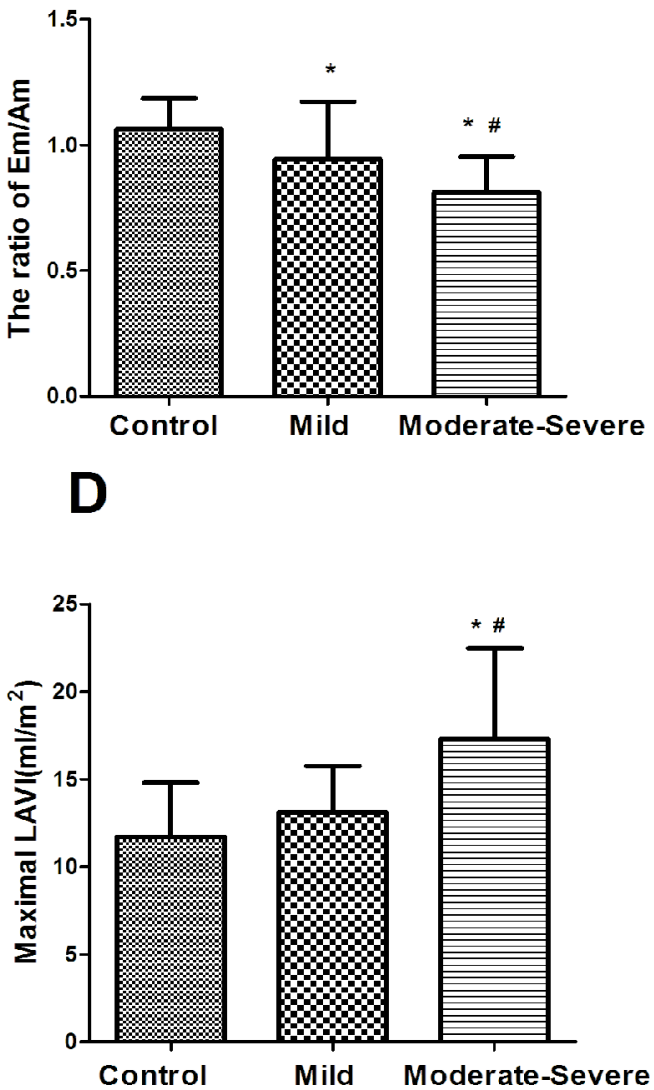

Figure 1: Left ventricular diastolic functional parameters in patients with OSA and controls.

Left ventricular diastolic functional parameters were measured by echocardiography. A) The ratio of early diastolic peak transmitral flow velocity (E) to peak early diastolic mitral annular velocity (Em); B) Em; C) The ratio of Em to peak late diastolic mitral annular velocity (Am); D) Maximal left atrial volume index (LAVI). Data are presented as mean $\pm S D$. ( $n=25$ in control group, $n=30$ in mild OSA group, $n=36$ in moderate-severe OSA group). *: $\mathrm{P}<0.05$, mild OSA and moderate-severe OSA groups vs. control; \#: $\mathrm{P}<0.05$, moderate-severe OSA group vs. mild OSA. 
Table 2: Correlations between $\mathrm{AHI}, \mathrm{ODI}$ and $\mathrm{PTX}$, left ventricular diastolic functional parameters in study population.

\begin{tabular}{|l|l|l|l|l|}
\hline \multicolumn{1}{|c|}{ AHI } & \multicolumn{3}{l|}{ ODI } \\
\hline & $\boldsymbol{r}$ & $\boldsymbol{P}$ & $\boldsymbol{r}$ & $\boldsymbol{P}$ \\
\hline PTX3 & 0.607 & 0.000 & 0.603 & 0.000 \\
\hline Em & -0.389 & 0.000 & -0.435 & 0.000 \\
\hline Em/Am & -0.436 & 0.000 & -0.458 & 0.000 \\
\hline E/Em & 0.334 & 0.000 & 0.378 & 0.000 \\
\hline Max LAVI & 0.483 & 0.000 & 0.445 & 0.000 \\
\hline
\end{tabular}

ODI: Oxygen Desaturation Index; AHI; Apnea Hypopnea Index; PTX3: Pentraxin 3; E: Early Diastolic Peak Transmitral Flow Velocity; Em: Peak Early Diastolic Mitral Annular Velocity; Am: Peak Late Diastolic Annular Velocity; Max LAVI: Maximal Left Atrial Volume Index.

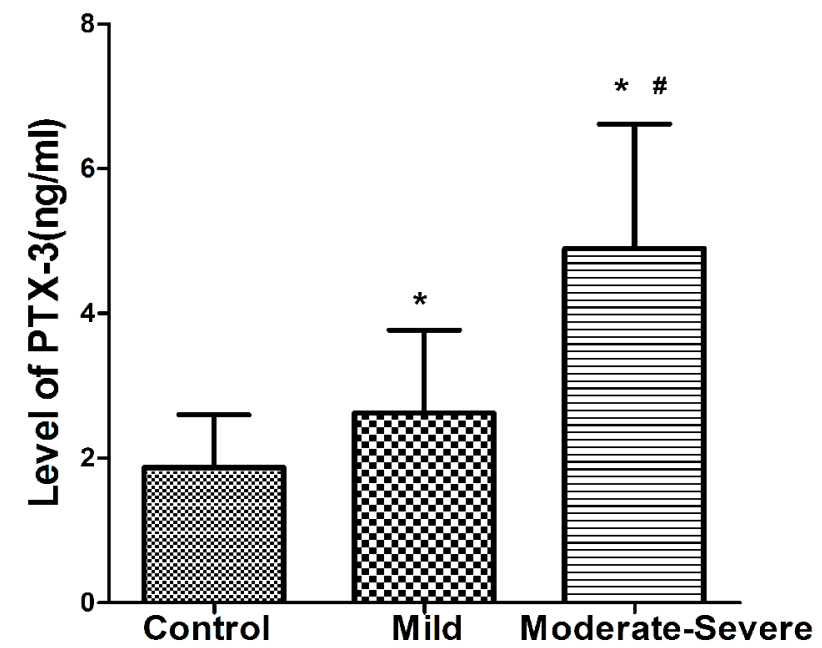

Figure 2: Level of PTX-3 in serum.

Pentraxin-3 (PTX-3) contents were measured by enzymelinked immunosorbent assay. Data are presented as mean \pm SD. $(n=25$ in control group, $n=30$ in mild OSA group, $n$ $=36$ in moderate-severe OSA group). ${ }^{*}: \mathrm{P}<0.05$, mild OSA and moderate-severe OSA groups vs. control; \#: $\mathrm{P}<0.05$, moderate-severe OSA group vs. mild OSA.

\section{Effects of OSA on left ventricular function}

As a well-established maker of left ventricular systolic function, LVEF was not significantly different among 3 groups (Table 1). Among the left ventricular diastolic functional parameters, there were no significant difference in the $E, A$, and $E / A$ ratio among 3 groups (Table 1 ). The Am was also not markedly different among 3 groups (Table 1). The E/Em ratio of OSA patients was significantly higher than that of the control group (Table 1 and Figure 1A). However, Em/ $A m$ ratio and Em were lower in patients with OSA than in the control group (Table 1, Figure 1B and Figure 1C). Furthermore, the maximal LAVI of the patients with moderate-severe OSA was significantly larger than that of the control group (Table 1 and Figure 1D). Em and $\mathrm{Em} / \mathrm{Am}$ ratio gradually decreased with the increase of OSA severity. However, the E/Em ratio gradually increased with the increase of OSA severity. In addition, there were negative correlation between the $\mathrm{Em} / \mathrm{Am}$ ratio, Em and ODI $(r=-0.458, r=-0.435, P<0.01)$, AHI $(r=-0.436, r=-0.389, P<0.01$ ) (Table 2). However, there were positive correlation between the $\mathrm{E} / \mathrm{Em}$ ratio, maximal LAVI and ODI $(r=0.378, r=0.445, P<0.01)$, AHI $(r=0.334, r=0.483, P<0.01$ ) (Table 2).

\section{Effects of OSA on plasma PTX-3}

In our study, serum levels of PTX-3 were significantly different among the 3 groups $(P<0.05)$, accompanying with the increase of OSA severity, PTX-3 gradually increased (Table 1 and Figure 2). Moreover, PTX-3 was positively correlated with $\mathrm{AHI}(r=0.607, P<0.01)$, and ODI $(r=0.603, P<0.01$ ) (Table 2).

\section{Correlation between PTX-3, and left ventricular diastolic functional parameters}

The Pearson correlation analysis demonstrated that PTX-3 was positively correlated with the E/Em ratio and maximal LAVI $(r=0.562, r=0.550, P<0.01)$ (Figure $3 \mathrm{~A}$ and Figure $3 \mathrm{~B}$ ). However, PTX-3 was negatively correlated with the Em/Am ratio and $\mathrm{Em}(r=-0.516, r$ $=-0.565, P<0.01$ ) (Figure $3 \mathrm{C}$ and Figure 3D).

\section{Discussion}

In the present study, the results demonstrated the appearance of early asymptomatic left ventricle dysfunction and signs of subclinical inflammation in patients with OSA. The left ventricle dysfunction observed in our study was characterized by LVDD (reduced $\mathrm{Em} / \mathrm{Am}$ ratio, reduced $\mathrm{Em}$, increased $\mathrm{E} / \mathrm{Em}$ ratio, increased maximal LAVI) and was more evident in patients with moderate-severe OSA. Levels of PTX-3 in serum significantly increased in OSA patients. There was a positive correlation between LVDD and serum PTX-3 level in OSA patients.

LVDD was an important cause of cardiovascular morbidity and was regarded as the initial pathophysiological step of cardiac abnormality [12]. Previous studies showed that ventricular diastolic dysfunction predicted the risk of the clinical atherosclerosis, hypertension and heart failure [4]. Some studies had described that OSA was an independent risk factor for the development of cardiac disorders. LVDD was common complication in OSA patients [13]. Alper Kepez, et al. [14] determined significantly decreased mitral E/A ratio and significantly increased left atrial diameters in OSA patients compared with control group. Recently study reported the presence of left ventricular hypertrophy, left atrial dilatation and LVDD in OSA patients without cardiovascular diseases [15]. Our studies showed that the ratio of $\mathrm{E} / \mathrm{Em}$ and maximal LAVI were higher in patients with moderate-severe OSA by means of echocardiography, a widely used non-invasive method. The ratio of $\mathrm{Em} / \mathrm{Am}$ and $\mathrm{Em}$ decreased in patients with OSA. However, there was no significant change in LVEF among all groups. These results suggested that impairment of left ventricular diastolic function was common in OSA patients with preserved systolic function. $\mathrm{CIH}$ induced by respiratory events in OSA patients could result in cardiovascular function disorders. In the present study, 
A

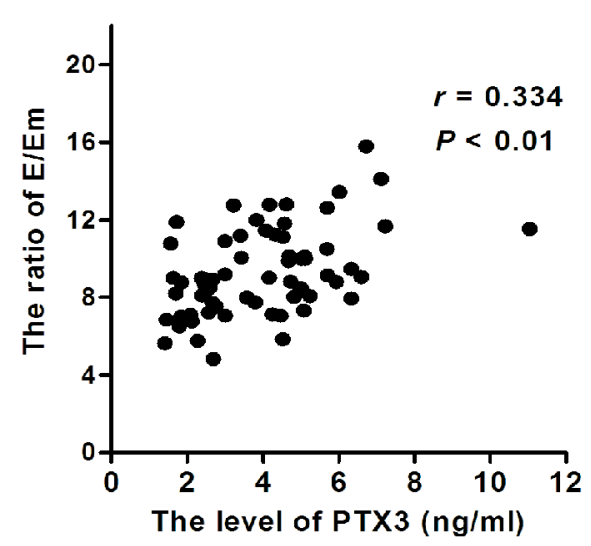

C

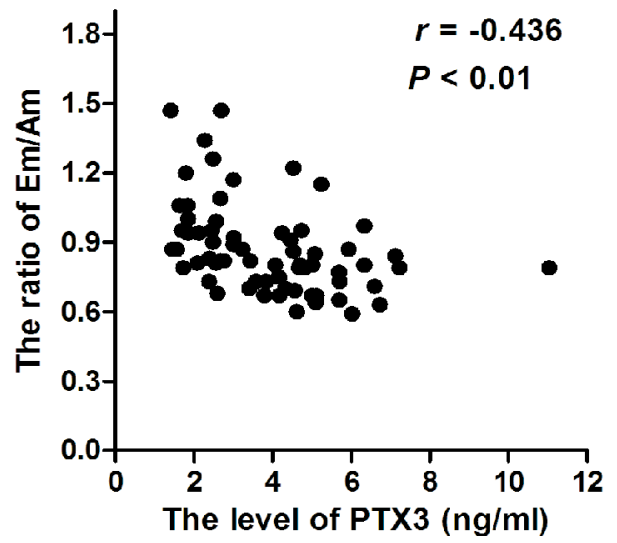

B

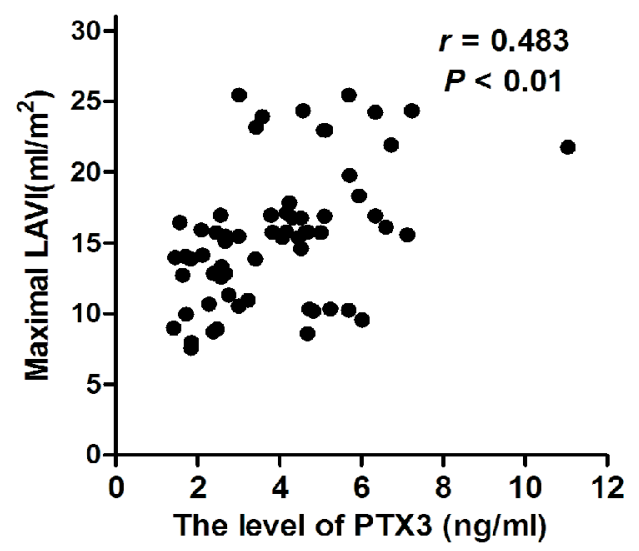

D

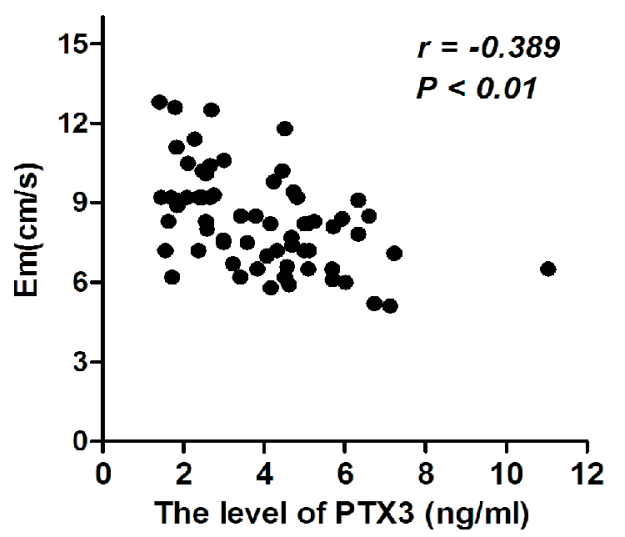

Figure 3: Correlation between PTX-3 and left ventricular diastolic functional parameters.

A) Correlations between the ratio of early diastolic peak transmitral flow velocity (E) to peak early diastolic mitral annular velocity (Em) and pentraxin-3 (PTX-3); B) Correlations between maximal left atrial volume index (LAVI) and PTX-3; C) Correlations between the ratio of Em to peak late diastolic mitral annular velocity (Am) and PTX-3; D) Correlations between Em and PTX-3.

our results showed that $\mathrm{AHI}$ and ODI in OSA patients were significantly higher than the control group. In addition, $\mathrm{AHI}$ and ODI were significantly correlated with LVDD assessed by $\mathrm{Em} / \mathrm{Am}$ ratio, Em, E/Em ratio and maximal LAVI in OSA patients, which suggested that $\mathrm{CIH}$ induced by OSA might directly contribute to LVDD.

Inflammatory was important for the pathogenesis and adverse prognoses of heart failure. The new inflammatory marker, PTX-3, was recently suggested as a predictor of poor prognosis of heart failure [16]. Importantly, PTX-3 was identified as an independent marker associated with the occurrence of LVDD among non-heart failure patients [11]. PTX-3 was produced in local inflammation and it was associated with LVDD. In the experimental study [11], Matsubara J, et al. showed that PTX-3 was produced in the coronary circulation of patients with heart failure with normal ejection fraction and non-heart failure with LVDD. The amount of PTX3 production in coronary circulation was positively correlated with levels of PTX-3 in systemic circulation. The increased secretion of PTX-3 in coronary circulation might prove its major role in the pathogenesis of LVDD. On the contrary, some studies $[17,18]$ showed that PTX-3 might play a protective role in the cardiovascular system by regulating the immunoinflammatory balance. Therefore, the increased PTX-3 would play a compensatory cardioprotective role at the activated inflammatory condition. However, in the present study, our results showed that levels of PTX-3 in serum significantly increased and was significantly correlated with asymptomatic LVDD assessed by echocardiography in OSA patients. PTX-3 might play an important role in OSA-associated vascular dysfunction [9]. Some studies showed the increased levels of ptx-3 in serum of OSA patients. A study conducted by Kasai, et al. showed that serum PTX-3 levels and arterial stiffness notably increased in OSA patients compared with control subjects, and continuous positive airway pressure treatment improved PTX-3 and arterial stiffness [9]. For that matter, our studies showed increased serum PTX-3 levels in OSA patients compared with control subjects, which was consistent with previous studies $[19,20]$. Moreover, PTX-3 was positively correlated 
with $\mathrm{AHI}$ and ODI. A significant positive correlation was also found between PTX-3 and the E/Em ratio and maximal LAVI in OSA patients, respectively. PTX-3was also negatively correlated with the Em/Am ratio and Em, respectively. The results showed that PTX-3 levels were markedly correlated with the presence of LVDD among OSA patients, and that PTX-3 was an important inflammatory marker to induce LVDD in OSA patients in our study, but not an inducible promoter. PTX-3, as an important inflammatory marker, could predict the risk of LVDD in patients with OSA. Further studies might need to estimate pathological roles of PTX-3 in OSA patients with LVDD.

In conclusion, OSA patients showed increment of pentraxin-3 and the presence of LVDD. Furthermore, PTX-3 and LVDD were independently related with OSA severity. PTX-3, Em, Em/Am, E/Em and maximal LAVI could be used as signs of early asymptomatic left ventricular myocardial dysfunction in patients with OSA.

\section{Acknowledgements}

This work was supported by grants from science and technology foundation projects of Hebei Province (Project Number: 18277786D).

\section{Author Contributions}

Jie-Ru Li and En-Sheng Ji conceived and designed the experiments. Jie-Ru Li, Xiu-Hua Gao, Ju-Qiang Han, Wen-Yan Luo, participated in the experimental studies. Jie-Ru Li and En-Sheng Ji wrote the paper.

\section{Sources of Support}

The project supported by science and technology foundation projects of Hebei Province (Project Number: 18277786D).

\section{References}

1. Sanna A (2017) Obstructive sleep apnea syndrome (OSAS): Continuous positive airway pressure (CPAP) therapy and other positive-pressure devices. Med Lav 108: 283-287.

2. Vrints $H$, Shivalkar $B$, Hilde $H$, Vanderveken $O M$, Hamans E, et al. (2013) Cardiovascular mechanisms and consequences of obstructive sleep apnoea. Acta Clin Belg 68: 169-178.

3. Kawata T, Daimon M, Miyazaki S, Ichikawa R, Maruyama $M$, et al. (2015) Coronary microvascular function is independently associated with left ventricular filling pressure in patients with type 2 diabetes mellitus. Cardiovasc Diabetol 14: 98.

4. Kane GC, Karon BL, Mahoney DW, Redfield MM, Roger VL, et al. (2011) Progression of left ventricular diastolic dysfunction and risk of heart failure. JAMA 306: 856-863.

5. Lin JC, Lin CL, Chen MC, Chang PJ, Chang ST, et al. (2015) Gout, not hyperuricemia alone, impairs left ventricular diastolic function. Arthritis Res Ther 17: 323-331.

6. Bavia L, Lidani KCF, Andrade FA, Sobrinho MIAH, Nisihara RM, et al. (2018) Complement activation in acute myocardial infarction: An early marker of inflammation and tissue injury? Immunol Lett 200: 18-25.
7. Floras JS (2018) Sleep apnea and cardiovascular disease: An enigmatic risk factor. Circ Res 122: 1741-1764.

8. Vilahur G, Badimon L (2015) Biological actions of pentraxins. Vascul Pharmacol 73: 38-44.

9. Kasai $\mathrm{T}$, Inoue $\mathrm{K}$, Kumagai $\mathrm{T}$, Kato M, Kawana $\mathrm{F}$, et al. (2011) Plasma pentraxin3 and arterial stiffness in men with obstructive sleep apnea. Am J Hypertens 24: 401-407.

10. Kanbay A, Kaya E, Büyükoğlan H, Kaya MG, Şimşek ZÖ, et al. (2015) Correlation between pentraxin-3 and endothelial dysfunction in obstructive sleep apnea syndrome. Ann Thorac Med 10: 199-203.

11. Matsubara J, Sugiyama S, Nozaki T, Sugamura K, Konishi $M$, et al. (2011) Pentraxin 3 is a new inflammatory marker correlated with left ventricular diastolic dysfunction and heart failure with normal ejection fraction. J Am Coll Cardiol 57: 861-869.

12. Bodez D, Damy T, Soulat-Dufour L, Meuleman C, Cohen A (2016) Consequences of obstructive sleep apnoea syndrome on left ventricular geometry and diastolic function. Arch Cardiovasc Dis 109: 494-503.

13. Bodez D, Lang S, Meuleman C, Boyer-Châtenet L, Nguyen $\mathrm{XL}$, et al. (2015) Left ventricular diastolic dysfunction in obstructive sleep apnoea syndrome by an echocardiographic standardized approach: An observational study. Arch Cardiovasc Dis 108: 480-490.

14. Kepez A, Niksarlioglu EY, Hazirolan T, Ranci O, Kabul HK, et al. (2009) Early myocardial functional alterations in patients with obstructive sleep apnea syndrome. Echocardiography 26: 388-396.

15. Aslan K, Deniz A, Cayli M, Bozdemir H, Sarica Y, et al. (2013) Early left ventricular functional alterations in patients with obstructive sleep apnea syndrome. Cardiol J 20: 519525.

16. Suzuki S, Takeishi Y, Niizeki T, Koyama Y, Kitahara T, et al. (2008) Pentraxin 3, a new marker for vascular inflammation, predicts adverse clinical outcomes in patients with heart failure. Am Heart J 155: 75-81.

17. Shimizu T, Suzuki S, Sato A, Nakamura Y, Ikeda K, et al. (2015) Cardio-protective effects of pentraxin 3 produced from bone marrow-derived cells against ischemia/ reperfusion injury. J Mol Cell Cardiol 89: 306-313.

18. Casula M, Montecucco F, Bonaventura A, Liberale L, Vecchié A, et al. (2017) Update on the role of Pentraxin 3 in atherosclerosis and cardiovascular diseases. Vascul Pharmacol 99: 1-12.

19. Ciccone MM, Scicchitano $P$, Zito A, Cortese F, Boninfante B, et al. (2014) Correlation between inflammatory markers of atherosclerosis and carotid intima-media thickness in obstructive sleep apnea. Molecules 19: 1651-1662.

20. Kim J, Gozal D, Bhattacharjee R, Kheirandish-Gozal L (2013) TREM-1 and pentraxin-3 plasma levels and their association with obstructive sleep apnea, obesity, and endothelial function in children. Sleep 36: 923-931. 